\title{
Whole-Body but Not Hepatic Knockdown of Chemerin by Antisense Oligonucleotide Decreases Blood Pressure in Rats
}

\author{
David J. Ferland, Bridget Seitz, Emma S. Darios, Janice M. Thompson, Steve T. Yeh, ${ }^{1}$ \\ Adam E. Mullick, ${ }^{1}$ and Stephanie W. Watts \\ Department of Pharmacology and Toxicology, Michigan State University, East Lansing, Michigan (D.J.F., B.S., E.S.D., J.M.T., \\ S.W.W.) and lonis Pharmaceuticals, Carlsbad, California (S.T.Y., A.E.M.)
}

Received October 13, 2017; accepted February 16, 2018

\begin{abstract}
Chemerin is an inflammatory adipokine positively associated with hypertension and obesity. The majority of chemerin derives from the liver and adipose tissue, however, their individual contributions to blood pressure are unknown. We began studying chemerin in the normal rat using antisense oligonucleotides (ASO) with whole-body activity (Gen 2.5 chemerin ASO) or liver-restricted activity (GalNAc chemerin ASO). We hypothesized that in normotensive male SpragueDawley rats, circulating chemerin is predominately liver-derived and regulates blood pressure. A dosing study of the Gen 2.5 chemerin ASO (with a scrambled control ASO) supported $25 \mathrm{mg} / \mathrm{kg}$ as the appropriate dose. GalNAc chemerin ASO was also assessed and used at $10 \mathrm{mg} / \mathrm{kg}$. Radiotelemetry monitored mean arterial pressure (MAP) for a 1-week baseline and weekly subcutaneous ASO injections for 4 weeks. Two
\end{abstract}

days after the final injection, animals were euthanized for tissue reverse transcription-polymerase chain reaction and chemerin Western analysis. Gen 2.5 chemerin ASO treatments reduced chemerin mRNA and protein in liver, retroperitoneal fat (RP), and mesenteric perivascular adipose tissue (mPVAT), as well as reducing protein in plasma. GalNAc chemerin ASO treatments reduced chemerin $\mathrm{mRNA}$ and protein in liver and chemerin protein in plasma but had no effect on expression in RP fat or mPVAT. Gen 2.5 chemerin ASO treatment reduced MAP compared with control ASO but was unchanged in animals receiving the GalNAc chemerin ASO. Although circulating chemerin is liver-derived, it does not play a major role in blood pressure regulation. Local effects of chemerin from fat may explain this discrepancy and support chemerin's association with hypertension and obesity.

\section{Introduction}

Chemerin is an adipokine often positively associated with inflammatory conditions like psoriasis (Albanesi et al., 2009), metabolic syndrome (Cheon et al., 2017), dyslipidemia, and hypertension (Zylla et al., 2017). Being an adipokine, chemerin is also positively associated with obesity (Chakaroun et al., 2012) and visceral adipose tissue in both rat and human models (Bozaoglu et al., 2007).

The Framingham Heart Study established the positive association between hypertension and adiposity (Garrison et al., 1987), but further analysis points toward visceral and retroperitoneal fat as the specific culprits in the pathology of obesity (Hall et al., 2015). This places chemerin production, and its propensity for inflammatory activity, in the very same place as the source of obesity-associated hypertension, the visceral adipose tissue. One component of this visceral fat depot is the mesenteric perivascular adipose tissue (mPVAT). These white adipocytes directly surround the mesenteric resistance vessels, which are thought to play a major role in the regulation of blood pressure (Christensen and Mulvany, 2001). The

This research was funded by the National Institutes of Health [Grant HL117847] and Ionis Pharmaceuticals, Carlsbad, CA.

${ }^{1}$ Current affiliation: Ionis Pharmaceuticals, Carlsbad, CA.

https://doi.org/10.1124/jpet.117.245456. proximity and possible paracrine release of chemerin from the mPVAT could explain some of the blood pressure effects of chemerin. Although there is a possible connection between PVAT and the etiology of hypertension, the role of the liver in this paradigm remains more of a mystery. It is well established that the primary producer of chemerin mRNA is the liver, with the second highest being the visceral adipocytes (Bozaoglu et al., 2007). This begs the question, "Which source of chemerin is more important to the association of chemerin with hypertension, liver or fat?"

To begin this investigation into the association of chemerin with hypertension, it was worthwhile to first inquire how the physiology of chemerin operates in the healthy normal rat. When isolated aorta, superior mesenteric artery (SMA), and mesenteric resistance vessels with a normal endothelium are exposed to chemerin, the contraction of the vessel is slight. However, when the vessel is contracted with phenylephrine, the vessel further contracts in response to chemerin to the same degree as when the endothelium is damaged or removed (Watts et al., 2013). These conclusions have been supported by other laboratories that noted the importance of the endothelium in protecting against chemerin-induced contraction (Neves et al., 2014). The normal SMA also has the ability to contract in a chemerin receptor-dependent manner when stimulated with an electrical field, a contraction that was

ABBREVIATIONS: ANOVA, analysis of variance; ASO, antisense oligonucleotide; GalNAc, $N$-acetylgalactosamine; MAP, mean arterial pressure; mPVAT, mesenteric perivascular adipose tissue; PBS, phosphate-buffered saline; RP, retroperitoneal; SMA, superior mesenteric artery. 
dependent on PVAT and the sympathetic nervous system (Darios et al., 2016). We have identified the mechanism of vascular contraction in normal animals as an operation through the smooth muscle cells themselves that works in a calcium-dependent manner (Ferland et al., 2017).

Antisense oligonucleotides (ASOs) represent a class of recent therapeutics that bind RNA through Watson-Crick pairing, which promotes RNA degradation or can have other modulatory effects such as redirecting splicing (Bennett et al., 2017). There are a variety of ASO modifications that enhance in vivo stability, distribution, and RNA binding affinity. For example, the (S)-constrained ethyl (cET; Gen 2.5) sugar modification improves RNA binding affinity, leading to improved in vivo activity in hepatic and extrahepatic tissue (Hung et al., 2013). Additionally, recent advances in targeted delivery using the trivalent $N$-acetylgalactosamine (GalNAc) conjugation have demonstrated improved targeting of the liver (Prakash et al., 2016). GalNAc conjugation on ASOs containing 2'-O-methoxyethyl sugar modification (MOE; Gen 2.0) have demonstrated robust liver activity, with little to no activity in adipose tissue (Mullick et al., 2017). Thus, to dissect the role of liver-derived-versus-extrahepatic chemerin, a GalNAc conjugated Gen 2.0 ASO with high specificity for reducing liver chemerin expression was compared with the Gen 2.5 ASO, which potently reduced liver and adipose chemerin expression.

Because chemerin influences contraction of normal rat aorta, SMA, and mesenteric resistance vessels (both with and without prior contraction with phenylephrine) and electrical field-stimulated contraction, we chose to begin our in vivo studies with normal Sprague-Dawley (SD) rats. We hypothesized that 1) a removal of chemerin protein from the body by decreased mRNA translation would reduce mean arterial pressure (MAP), 2) the degree of MAP reduction would be correlated to the amount of circulating chemerin, and 3) the liver would be the major contributor to the circulating chemerin stores. Using in vivo pharmacology and constant cardiovascular monitoring, we were able to test if knockdown of chemerin by the liver-specific ASO (GalNAc Gen 2.0) or whole-body ASO (Gen 2.5) could reduce MAP. Western blotting and polymerase chain reaction allowed us to determine the relative associations between the terminal tissue or circulating chemerin and the short-term effect on MAP.

\section{Materials and Methods}

Animal Care. All procedures that involved animals were performed in accordance with the institutional guidelines on animal use committee of Michigan State University and the NIH Guidelines on Use of Laboratory Animals. Animals were housed singly and maintained on a $12 / 12$ light/dark cycle at a temperature of $22-25^{\circ} \mathrm{C}$. Normal male Sprague-Dawley rats (225-300 g; Charles River Laboratories, Inc., Portage, MI) were used.

ASO Synthesis. Synthesis of the Gen 2.0 and 2.5 antisense oligonucleotides (Seth et al., 2008) and GalNAc ASOs (Prakash et al., 2016) were described previously. In vitro activity screens followed by in vivo activity and tolerability screens were used to identify leads. The following are lists of the scrambled, GalNAc Gen 2.0 and Gen 2.5 ASOs used in this study:

Scrambled $\left(5^{\prime}-3^{\prime}\right)$ : GGCCAATACGCCGTCA

GalNAc Gen $2.0\left(5^{\prime}-3^{\prime}\right)$ : GalNAc-ACAGTTTTATTAGCCTGGAG

Gen $2.5\left(5^{\prime}-3^{\prime}\right)$ : GTTTTATTAGCCTGGA
ASOs were diluted in sterile phosphate-buffered saline (PBS) to their final concentration (described below).

Radiotelemetry and In Vivo Pharmacology. Rats were implanted with a femoral catheter connected to a subcutaneously implanted radiotelemeter. Early studies used C-40s (DSI, New Brighton, MN) but were replaced with HD-S10s (DSI) for the added capability of measuring core body temperature. After 5 days of recovery, baseline cardiovascular measures were recorded every 10 minutes for 10 seconds over 1 week. Weekly subcutaneous injections were performed under anesthesia (1-2\% isoflurane) for 4 weeks. Physiologic measures were sampled every 10 minutes for 10 seconds for the duration of the experiment and averaged daily for statistical analysis and graphical representation.

Gen 2.5 Chemerin ASO Dosing Study. After implantation of radiotelemetry, rats were administered a scrambled control ASO or Gen 2.5 chemerin ASO. Both control ASO and Gen 2.5 chemerin ASO were administered at $25,12.5$, or $6.25 \mathrm{mg} / \mathrm{kg}$. Doses were given subcutaneously on days $0,7,14$, and 19 with tissue and blood collection on day 21.

Gen 2.5 Chemerin and GalNAc Chemerin ASO Blood Pressure Studies. After implantation of radiotelemetry, rats received subcutaneous injection of PBS (average volume of ASO injections), control ASO (25 mg/kg), Gen 2.5 chemerin ASO (25 mg/ $\mathrm{kg}$ ), or GalNAc chemerin ASO (10 mg/kg) on days 0, 7, 14, and 19 . Animals were sacrificed under anesthesia (1-2\% isoflurane) by pneumothorax. The entire retroperitoneal fat pad was dissected away from the posterior abdominal wall and the mPVAT was removed in bulk from the base of the SMA without collecting any of the mesenteric resistance vessels. Tissues were weighed and collected for mRNA and protein analysis. Blood was also collected for plasma. All samples were stored either at $-80^{\circ} \mathrm{C}$ or under dry-ice until isolation of mRNA or protein. Researchers performing the mRNA and protein analysis were initially blinded to the treatment conditions of the tissue.

Real-Time Quantitative PCR. Quantitative real-time reverse transcription-polymerase chain reaction (qPCR) mRNA analysis was performed with TaqMan primer probes (Applied Biosystems; Thermo Fisher Scientific, Foster City, CA). Total RNA was extracted from whole tissue with the RNeasy RNA isolation kit (Qiagen, Valencia, CA). Samples (50 ng total RNA) were subjected to qPCR analysis with commercial reagents (Invitrogen; Thermo Fisher Scientific, Carlsbad, CA) and analyzed with the ABI StepOne Plus Sequence Detector (Applied Biosystems). TaqMan primers and probe for chemerin are as follows: forward sequence, CAGGAGATCGGTGTGGACAGT; reverse sequence, GAGCTTAAATTCC-AGCCTCACAA; probe sequence, TGATGACCTGTTCTTCTCAGCTGGCACCX. The PCR probes were labeled with 5 '-FAM (a 6 - carboxyfluorescein reporter) and $3^{\prime}$-TAMRA [a 5(6)-carboxytetramethyl rhodamine quencher]. After 40 amplification cycles, absolute values were obtained with SDS analysis software (Applied Biosystems). Values were normalized to total RNA via RiboGreen measurement (Invitrogen). The use of total RNA as a calibration control has been validated (Hashimoto et al., 2004).

Western Analysis. Blood was centrifuged for 20 minutes at $4^{\circ} \mathrm{C}$ and $2000 \mathrm{rpm}$. Plasma was collected and diluted 1:25 before performance of a bicinchoninic acid assay for total protein (cat. no. BCA1; MilliporeSigma, St. Louis, MO). Fat pads were homogenized in an Bead Ruptor $24(5.65 \mathrm{~m} / \mathrm{s}, 2$ cycles, 30-second cycles, 30 seconds between cycles, $6^{\circ} \mathrm{C}$; Omni International, Kennesaw, GA). Onehundred micrograms of protein was added to a $15 \%$ polyacrylamide gel and run at $120 \mathrm{~V}$. Protein was transferred to a Immobilon FL PVDF Membrane (cat. no. IPFL00010; MilliporeSigma, Billerica, MA) for 1 hour at 100 V. Blots were dried, Total Protein Stain (cat. no. 92611011; Li-Cor, Lincoln, NE) was added (if applicable) and reverted, then blocked with Chick Egg Ovalbumin for 3 hours. Chemerin antibody (1:1000; cat. no. 112520; Abcam, Cambridge, MA) was incubated for 48 hours at $4^{\circ} \mathrm{C}$ and the secondary antibody $(1: 1000$; IRDye 800 anti-Mouse, cat. no. 926-32210; Li-Cor) was incubated for 1 hour at $4^{\circ} \mathrm{C}$. Transferrin was used as a loading control (1:1000; cat. no. 82411; Abcam) with an IRDye 680LT secondary (1:1000; anti-rabbit, 
cat. no. 926-68021; Li-Cor) for plasma, and Total Protein Stain was the loading control for tissues. Total protein quantification is becoming the preferred method of performing a loading control, because it does not rely on the expression of one protein and can more ubiquitously control for expression changes over a large range of tissue types (Eaton et al., 2013). Blots were visualized using the Odyssey FC and CLx Infrared Imaging and quantified using Image Studio (5.2.5; Li-Cor).

Data Analyses and Statistics. All mRNA data were compiled, normalized to total RNA, then normalized to an average of all controls (PBS and scrambled control ASO). All percentages of mRNA expression were compared with this averaged control. Protein was quantified then normalized to loading controls (total protein or transferrin). If there were comparisons made between different blots, samples were further normalized to their respective PBS control. All percentages of chemerin protein expression were compared with an average of the control ASO measurement. Although Western images may have brightness or contrast enhanced as a whole to visualize bands, it does not change the quantification by the Li-Cor Image Studio. All comparisons of significance were performed in Prism 7 (vers. 7.0c; Graph Pad, La Jolla, CA) using one-way analysis of variance (ANOVA) with a Sidak correction for multiple comparisons. Data are reported as mean \pm S.E.M. In vivo data were analyzed by two-way ANOVA (treatment and time) comparing the mean of each treatment with the others using a Sidak correction for multiple comparisons.

\section{Results}

Gen 2.5 Chemerin ASO Must Be Dosed at $25 \mathrm{mg} / \mathrm{kg}$ for Effective Knockdown. A small experiment assessed the ability of the Gen 2.5 chemerin ASO to knock down chemerin mRNA concentrations at weakly consistent doses of $25,12.5$, and $6.25 \mathrm{mg} / \mathrm{kg}$, comparing them all to the scrambled control ASO at the same dose. Chemerin mRNA expression in the liver (Fig. 1A) was completely reduced with the $25-\mathrm{mg} / \mathrm{kg}$ dose compared with the control ASO. Expression with 12.5 - and $6.25-\mathrm{mg} / \mathrm{kg}$ dosing of Gen 2.5 chemerin ASO rose to $3.3 \% \pm 0.3 \%$ and $9.0 \% \pm 2.3 \%$, respectively. Likewise, retroperitoneal (RP) fat (Fig. 1B) exhibited a complete reduction in chemerin expression at $25 \mathrm{mg} / \mathrm{kg}$ with expression increasing to $16.0 \% \pm 3.5 \%$ and $57.3 \% \pm 2.7 \%$ with 12.5 and $6.25 \mathrm{mg} / \mathrm{kg}$, respectively. Chemerin expression from the mPVAT (Fig. 1C) was variable but the protein and mRNA results from later studies (Fig. 3, E and F) indicate that the ASO is working and effective in the mPVAT at $25 \mathrm{mg} / \mathrm{kg}$. Circulating chemerin protein (Fig. 1D) was also measured in this dosing study with complete knockdown of chemerin at the $25 \mathrm{mg} / \mathrm{kg}$ dose and levels rising to $6.0 \% \pm 1.5 \%$ and $10.3 \% \pm$ $2.7 \%$ in the $12.5-$ and $6.25-\mathrm{mg} / \mathrm{kg}$ doses, respectively. Thus, $25 \mathrm{mg} / \mathrm{kg}$ was used as the dose of the chemerin ASO in vivo.

The goal of dosing the GalNAc chemerin ASO was to accomplish the complete knockdown of the liver as seen in the Gen 2.5 chemerin ASO, at the same time maintaining chemerin expression in the fat pads. GalNAc-tagged ASOs have an increased liver potency of 10- to 30-fold (Prakash et al., 2016). On the basis of this information, preliminary activity studies, and the studies we will share in Fig. 3, a dose of $10 \mathrm{mg} / \mathrm{kg}$ was used and proven effective.

Minimal Off-Target Effects with a 4-Week Chemerin ASO Regimen. Using established doses of Gen 2.5 chemerin ASO and GalNAc chemerin ASO, the effects of chemerin on physiologic parameters outside of MAP were minimal. There was no change in final body weight (Fig. 2A) among any of the treatment groups. In addition, there was no difference in the total weight of the RP fat (Fig. 2B) or heart (Fig. 2E). The kidney showed a slight increase compared with GalNAc chemerin ASO treatment (Fig. 2F), but because there was no increase compared with control ASO or Gen 2.5 chemerin ASO, it is not considered physiologically significant with respect to chemerin. The only differences in organ weight associated with ASOs that modified chemerin were observed with liver (Fig. 2C) and spleen (Fig. 2D). Both exhibited differences between the control groups (PBS and control ASO) and groups in which chemerin was removed (Gen 2.5 and GalNAc). Spleen weight increased 2.0-fold when treated against chemerin compared with controls.

Whole-Body Removal of Chemerin Lowers Blood Pressure. Gen 2.5 chemerin ASO at $25 \mathrm{mg} / \mathrm{kg}$ significantly reduced chemerin mRNA expression in the liver (Fig. 3A), RP fat (Fig. 3C), and mPVAT (Fig. 3E) down to $0.5 \% \pm 0.1 \%, 3.9 \%$ $\pm 0.4 \%$, and $30.7 \% \pm 9.7 \%$ of control levels, respectively. More importantly, this reduction in chemerin mRNA led to a proportional reduction in chemerin protein expression to $2.7 \% \pm 0.5 \%$ in liver (Fig. 3B), $0.8 \% \pm 0.7 \%$ in RP fat (Fig. 3D),
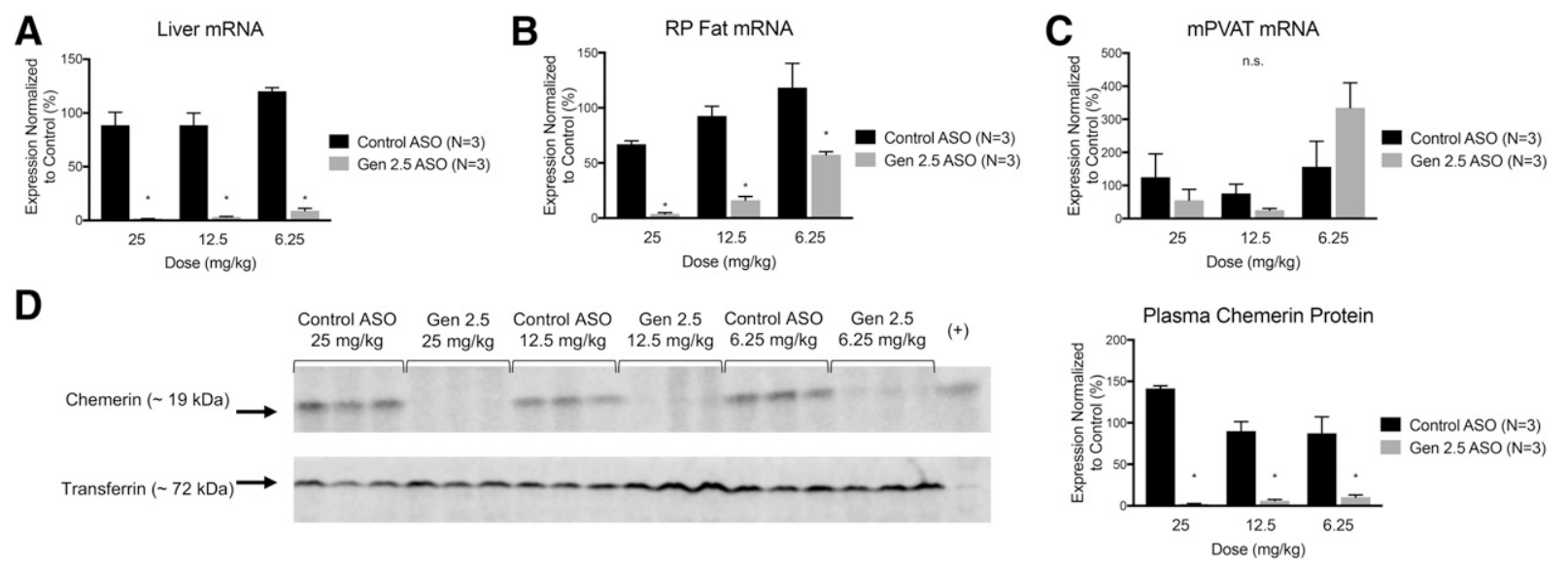

Fig. 1. Chemerin mRNA measures of liver (A), retroperitoneal (RP) fat (B), and mesenteric perivascular adipose tissue (C) along with plasma chemerin (D) to determine the optimum dosing scheme for Gen 2.5 chemerin ASO (gray bars) against a scrambled control ASO (black bars). Amount of RNA was first normalized to total RNA then normalized to an average of all controls, making a percent. Densitometry data were normalized to transferrin. $* P<$ $0.05 ;$ n.s., no statistical significance by One-Way ANOVA with a Sidak Correction. Western blots are representative samples of the included bar graph. $(+)$ represents the liver positive control separate from all treatments. Arrows indicate the levels of the indicated molecular weights. 

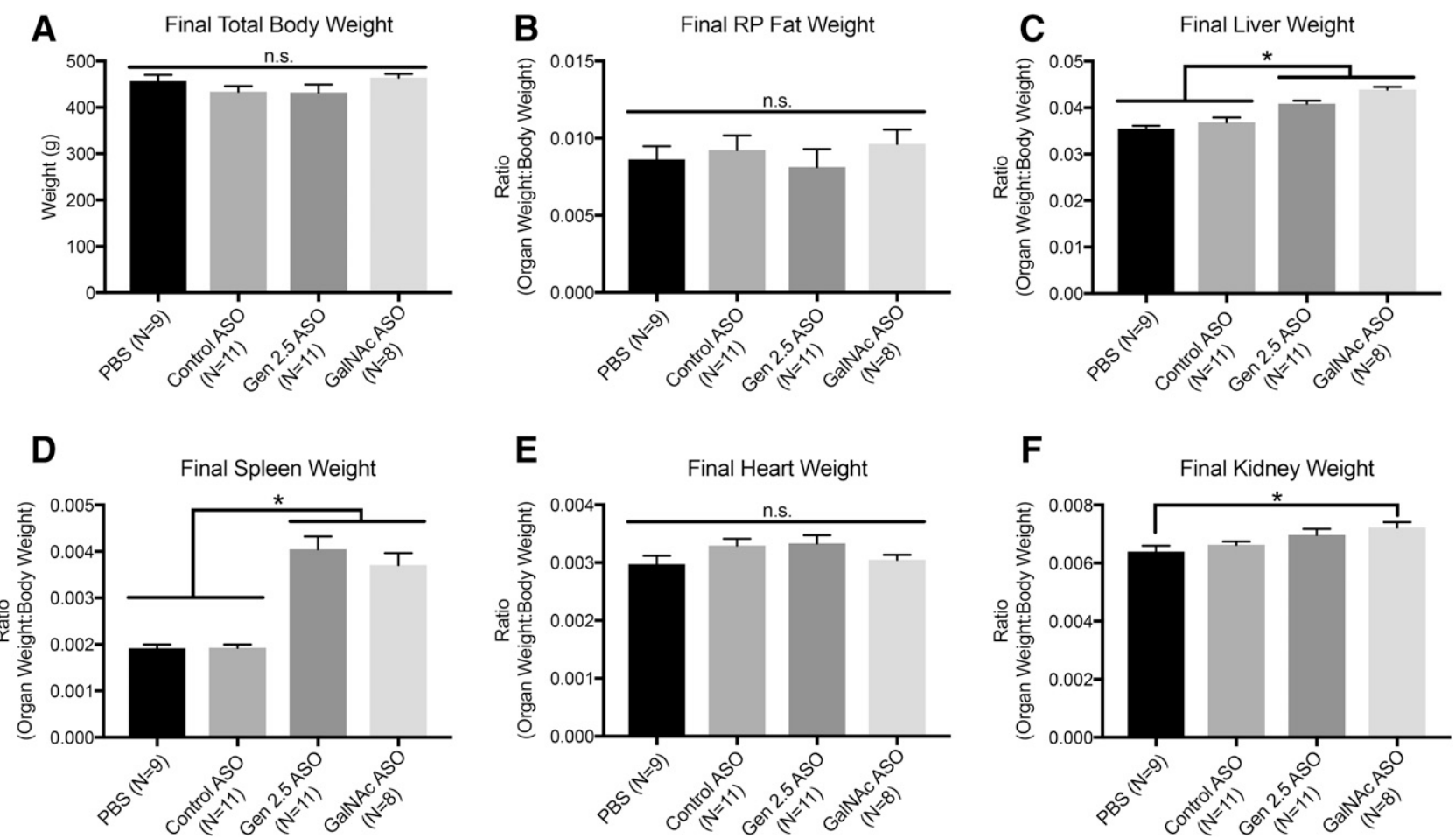

Fig. 2. Final weights of total mass (A), retroperitoneal (RP) fat (B), liver (C), spleen (D), heart (E), and kidney (F) after four injections of PBS, scrambled control ASO, Gen 2.5 chemerin ASO, or GalNAc chemerin ASO. All individual organs were normalized to their total body weight to give a ratio. ${ }^{*} P<$ $0.05 ;$ n.s., no statistical significance by one-way ANOVA with a Sidak correction.

and $6.0 \% \pm 1.1 \%$ in mPVAT (Fig. $3 \mathrm{~F}$ ). Representative blots are shown on the left of Fig. 4, and densitometry and normalization are shown on the right. Using the Gen 2.5 chemerin ASO, circulating levels of chemerin were also undetectable (95\% CI -0.02 to 0.24 ; Fig. 4 ).

With this systemic loss of chemerin, MAP was reduced to a nadir of $7 \pm 2.1 \mathrm{mmHg}$ within 48-72 hours after each injection (Fig. 5A). Among the Gen 2.5 ASO animals, there was no significant change in heart rate (Fig. 5B) but there was a reflexive temperature rise with any chemerin ASO treatment (Gen 2.5 and GalNAc; Fig. 5C).

Removal of Liver Chemerin Alone Does Not Show the Same Blood-Lowering Effects. In treatment with an ASO modified to specifically deliver its cargo to the liver (GalNAc), the 10-mg/kg dose maintained complete knockdown of liver chemerin mRNA (Fig. 3A) and protein (Fig. 3B). RP fat was not significantly affected by GalNAc chemerin ASO either in mRNA (Fig. 3C) or protein expression (Fig. 3D). Although chemerin mRNA was modestly reduced in the mPVAT with GalNAc chemerin ASO (Fig. 3E), its levels were significantly increased from Gen 2.5 chemerin ASO levels $(P=0.01)$. Likewise, the chemerin protein expression in mPVAT was not significantly reduced from control (Fig. 3F). Even with the fat depots expressing higher levels of chemerin, plasma chemerin levels were still reduced by $90 \% \pm 5 \%$ by the GalNAc chemerin ASO (Fig. 4). However, when MAP of the GalNAc chemerin ASO-treated animals was measured, there was no significant reduction in MAP (Fig. 5A). As with Gen 2.5 ASO, rats treated with GalNAc chemerin ASO did not show a change in heart rate (Fig. 5B) but did show spikes in temperature with injections (Fig. 5C). A liver-effective dose of GalNAc chemerin ASO at $10 \mathrm{mg} / \mathrm{kg}$ was confirmed by the knockdown of chemerin from liver and maintenance of chemerin expression and protein in the RP fat and mPVAT (Fig. 3).

\section{Discussion}

The literature of chemerin promotes two major roles for this molecule. First, chemerin is a chemokine with the ability to promote chemotaxis of dendritic cells (Zabel et al., 2005), natural killer cells (Della Chiesa et al., 2005), macrophages (Wittamer et al., 2003), and T lymphocytes (Samson et al., 1998). Second, chemerin is an adipokine (Goralski et al., 2007) with the ability to promote blood vessel formation (Bozaoglu et al., 2010). This dichotomy seems to be supported by the two classes of major producers of chemerin: liver and fat. Our goal was to take the first step toward understanding the role of chemerin, ultimately in adiposity-associated models of hypertension, by performing these critical studies in the normal rat.

The Liver Provides the Majority of Circulating Chemerin. When chemerin mRNA expression by the liver is virtually abolished (Fig. 3, A and $\mathrm{B}$ ) and adipose tissue expression remains high (Fig. 3, C-F), the plasma chemerin levels are drastically reduced (Fig. 4). This not only supports previous findings that the liver is the primary producer of chemerin mRNA (Bozaoglu et al., 2007), but is also the first demonstration that the majority of chemerin protein in the plasma of the rat is derived from the liver.

The role of the liver in promoting circulating chemerin levels supports the role of chemerin as a chemokine. Our understanding of the liver as an organ that regulates immune responses and inflammation is expanding. The liver is a highly perfused organ exposed to a variety of toxins and insults (especially from the gastrointestinal system) and other more 

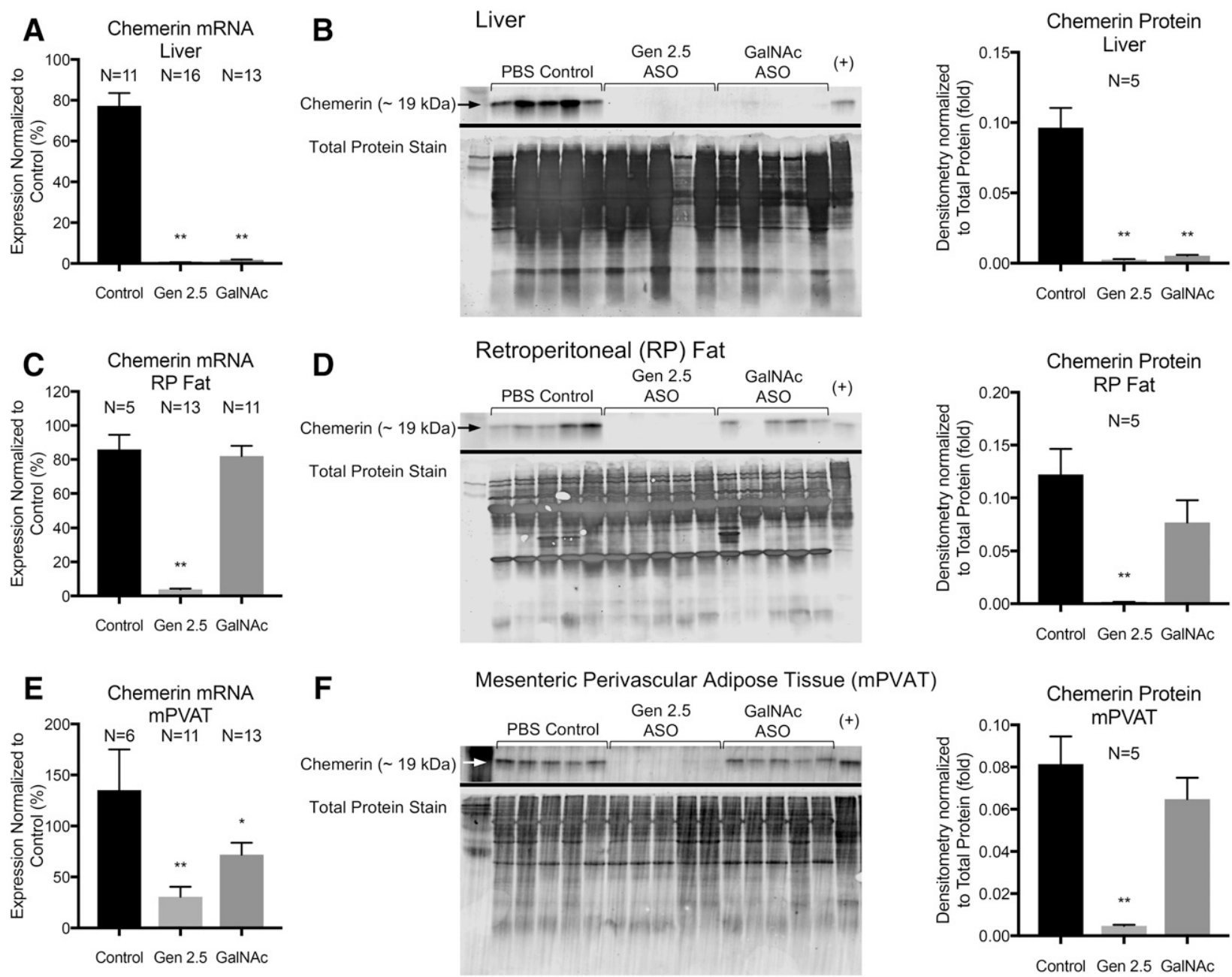

Fig. 3. Final measurements of chemerin mRNA and protein from liver (A and B), retroperitoneal (RP) fat (C and D), and mesenteric perivascular adipose tissue (E and F) after four treatments of PBS, Gen 2.5 chemerin ASO, or GalNAc chemerin ASO. Amount of RNA was first normalized to total RNA then normalized to an average of all controls, making a percent. Densitometry was normalized to total protein. ${ }^{*} P<0.05$; $* * P<0.01$ by one-way ANOVA with a Sidak correction. Western blots are representative samples of the included bar graph. (+) represents the liver positive control separate from all treatments. Arrows indicate the levels of the indicated molecular weights.

must manage healthy inflammation and other more hazardous stimuli (Robinson et al., 2016). The prominent association of the liver with circulating chemerin uncovered by this study indicates that chemerin may have a role to play in this evolving story of the liver as an immunomodulatory organ.

Both the liver and spleen had increased final weights when treated with both the Gen 2.5 chemerin ASO and GalNAc chemerin ASO (Fig. 2, C and D). Although ASOs can cause tissue hypertrophy, the treatments that caused a difference were those that targeted chemerin mRNA. Because the control ASO did not cause a spleen enlargement, the hypertrophy most probably is a result of the loss of chemerin and not an off-target effect of the ASO. Future studies will have to assess the microscopic and histologic changes of these two organs, but we can reasonably assume that because this hypertrophy also happened modestly in the liver of the GalNAc chemerin ASO-treated animal, the hypertrophy is related to liver function or plasma chemerin levels. The hypertrophy of the spleen was prominent in these studies and highlights a possible new role of chemerin in the body.

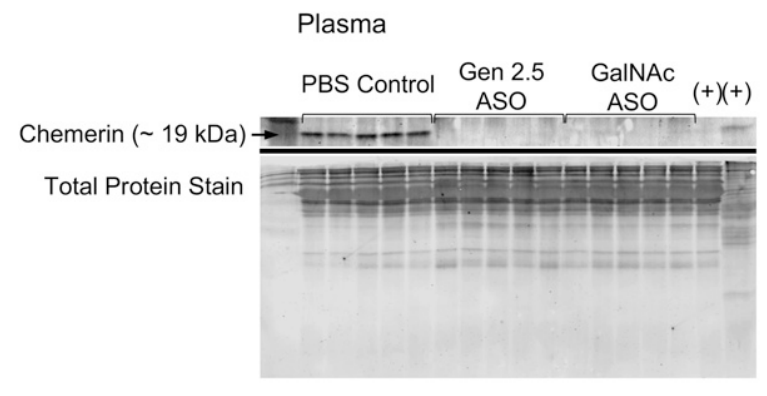

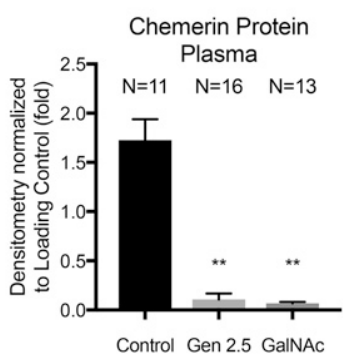

Fig. 4. Plasma chemerin is reduced after treatment with both Gen 2.5 chemerin ASO and GalNAc chemerin ASO. Densitometry was normalized to the loading control (either transferrin or total protein) then normalized to their respective PBS control for comparison between blots. $* * P<0.01$ by one-way ANOVA with a Sidak correction. Western blots are representative samples of the included bar graph. (+) represents the liver positive control separate from all treatments. Arrows indicate the levels of the indicated molecular weights. 

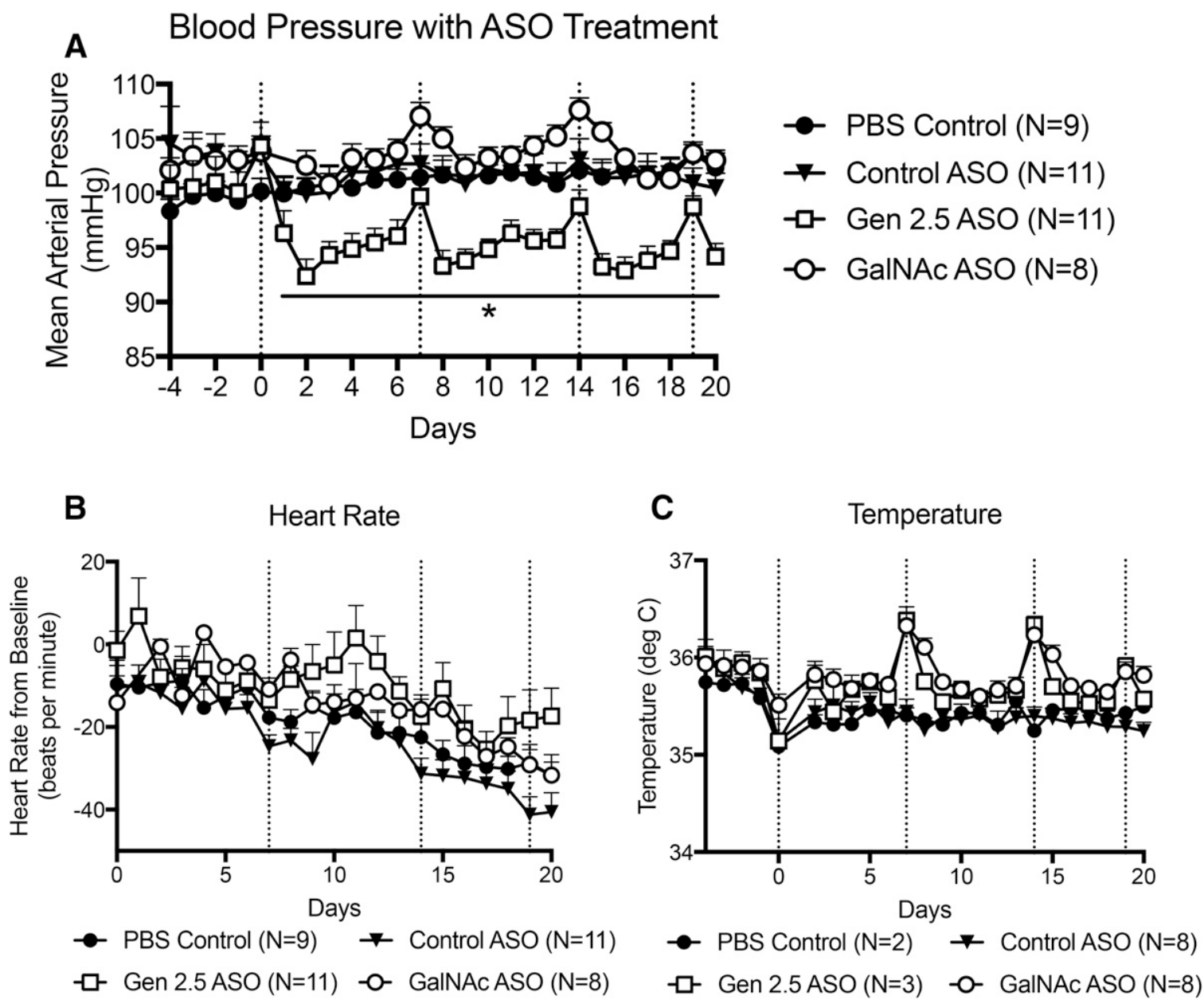

Fig. 5. Mean arterial blood pressure (A), heart rate (B), and temperature (C) collected by radiotelemetry during a 5-day baseline and 20-day treatment with four injections on days $0,7,14$, and 19 (dotted lines). ${ }^{*} P<0.05$ by two-way ANOVA with Sidak correction comparing the entire datasets. Baseline measures for heart rates: PBS control, $383 \pm 7$ beats/min; control ASO, $389 \pm 7$ beats/min; Gen 2.5 chemerin ASO, $379 \pm 8$ beats/min; and GalNAc chemerin ASO, $362 \pm 9$ beats $/ \mathrm{min}$. The baseline period (days -4 to -1 ) is not shown because it was used to calculate the subsequent values.

Blood Pressure Is Locally Regulated by Chemerin. Although the liver may support chemerin as a chemokine and provide circulating stores of the protein, it is not significantly involved in blood pressure regulation. On the basis of our initial hypothesis that the liver and circulating chemerin levels contribute to blood pressure regulation, we would have expected a proportional change between plasma chemerin levels and a lowering of MAP. With the GalNAc chemerin ASO, MAP remained at control levels (Fig. 5A), whereas plasma chemerin levels still dropped by $90 \%$ (Fig. 4). By contrast, MAP reduction in the whole-body knockdown of chemerin (Fig. 5A) demonstrates that chemerin still has a role to play in blood pressure regulation. This is in accordance with previous in vitro studies on blood vessel contractility (Watts et al., 2013; Ferland et al., 2017). A 7-mmHg fall in MAP with the whole-body chemerin ASO may seem modest, but this reproducible indication of blood pressure control by chemerin in a normal SD rat offers promise that continued study will demonstrate an interaction between chemerin and blood pressure. Future studies will focus on the effects of chemerin in hypertensive and obese rat models.

The most reasonable explanation for the reduction in MAP outside of circulating chemerin levels lies with the adipose tissue. First, chemerin is an adipokine. Second, among all organs in the body, the liver and white adipose are the primary producers of chemerin. Last, fat pads, where chemerin production is regulated (RP and mPVAT), are also implicated as important contributors to the pathology of hypertension. The MAP with ASO treatment does not seem to be affected by other cardiovascular factors like reductions in heart rate (Fig. $5 \mathrm{~B}$ ) or cardiac hypertrophy (Fig. 2E).

The maintenance of body weight across the treatments (Fig. 2A) along with the specific maintenance of $R P$ fat pad weights (Fig. 2B) supports a conclusion that Gen 2.5 chemerin ASO given over a 4-week period did affect the adipogenesis of this adipokine but still affected the secretion of the cells. Although chemerin secreted by the fat can certainly make its way into the circulation, the proportional loss in plasma chemerin protein and MAP between Gen 2.5 chemerin ASO and GalNAc chemerin ASO (Figs. 4 and 5) does not match. As such, the output of these adipocytes may also have a paracrine-like effect on the vascular beds they surround and protect.

Limitations. The ASO technology was necessary and invaluable for its ability to help us manipulate chemerin protein expression in vivo. However, there are currently no known ways to specifically target an ASO to adipocytes. As a result, 
we can draw direct conclusions about the function of the liver and chemerin but only inferences about the role of fat. This study can only conclude that the loss in MAP is the result of chemerin production in sites outside the liver. However, this study provides (indirect) support for the role of chemerin and fat in regulation of blood pressure. Future in vivo studies will have to approach this association directly, but the technology to do so does not currently exist.

Whereas chemerin mRNA expression in the RP fat (Fig. 1B) was reduced to $67 \% \pm 3 \%$ with $25 \mathrm{mg} / \mathrm{kg}$ control ASO and chemerin protein seemed to increase to $141 \% \pm 3 \%$ in the plasma (Fig. 1D) with $25 \mathrm{mg} / \mathrm{kg}$ control ASO, it is the complete reduction in chemerin expression that comes with the Gen 2.5 chemerin ASO at $25 \mathrm{mg} / \mathrm{kg}$ that is necessary for drawing conclusions about chemerin and other physiologic measures. Even though these effects of the control ASO may be of therapeutic concern, we do not make any claims in this study about the ideal therapeutic dosing - only the effective dose for chemerin knockdown. The increase of chemerin mRNA expression with the $25 \mathrm{mg} / \mathrm{kg}$ dose of control ASO did not change any other physiologic parameters, such as MAP, temperature, or heart rate (Fig. 5). As such, the apparent increase in chemerin mRNA with control ASO is an acceptable event to accomplish the complete knockdown of chemerin with the whole-body ASO.

These studies were performed on normotensive, healthy rats. Future studies will apply this technology and method to models of hypertension and obesity to observe if these changes in blood pressure are conserved or exacerbated in these pathologic states. Although we cannot use the present study to make any direct conclusions about chemerin in hypertension or obesity, linking chemerin to blood pressure and suggesting that blood pressure control is dependent on adipose tissue is an important stepping stone toward connecting chemerin to pathology.

\section{Conclusions}

Understanding the responsibilities of liver and fat in the biologic mechanisms of chemerin is important for reasons that are expanding and evolving, but the specific association of chemerin and blood pressure should focus on the fat. Thanks to the improving technology of ASOs, we were able to assess the role of chemerin in vivo in a way that efficiently targeted specific organs. We demonstrated that chemerin continues to be important in the world of blood pressure regulation, but the mechanism by which it accomplishes this regulation may be unconventional. All signs point toward chemerin having a local effect on the vasculature through the fat.

\section{Authorship Contributions}

Participated in research design: Watts, Mullick, Seitz, Ferland.

Conducted experiments: Seitz, Darios, Thompson, Yeh, Ferland.

Contributed new reagents or analytic tools: Mullick, Yeh.

Performed data analysis: Seitz, Ferland, Yeh, Mullick. Watts.

Wrote or contributed to the writing of the manuscript: Ferland,

\section{References}

Albanesi C, Scarponi C, Pallotta S, Daniele R, Bosisio D, Madonna S, Fortugno P, Gonzalvo-Feo S, Franssen JD, Parmentier M, et al. (2009) Chemerin expression marks early psoriatic skin lesions and correlates with plasmacytoid dendritic cell recruitment. J Exp Med 206:249-258.
Bennett CF, Baker BF, Pham N, Swayze E, and Geary RS (2017) Pharmacology of antisense drugs. Annu Rev Pharmacol Toxicol 57:81-105.

Bozaoglu K, Bolton K, McMillan J, Zimmet P, Jowett J, Collier G, Walder K, and Segal D (2007) Chemerin is a novel adipokine associated with obesity and metabolic syndrome. Endocrinology 148:4687-4694.

Bozaoglu K, Curran JE, Stocker CJ, Zaibi MS, Segal D, Konstantopoulos N, Morrison S, Carless M, Dyer TD, Cole SA, et al. (2010) Chemerin, a novel adipokine in the regulation of angiogenesis. J Clin Endocrinol Metab 95:2476-2485.

Chakaroun R, Raschpichler M, Klöting N, Oberbach A, Flehmig G, Kern M, Schön MR, Shang E, Lohmann T, Dreßler M, et al. (2012) Effects of weight loss and exercise on chemerin serum concentrations and adipose tissue expression in human obesity. Metabolism 61:706-714.

Cheon DY, Kang JG, Lee SJ, Ihm SH, Lee EJ, Choi MG, Yoo HJ, and Kim CS (2017) Serum chemerin levels are associated with visceral adiposity, independent of waist circumference, in newly diagnosed type 2 diabetic subjects. Yonsei Med $J$ 58: 319-325.

Christensen KL and Mulvany MJ (2001) Location of resistance arteries. J Vasc Res 38:1-12.

Darios ES, Winner BM, Charvat T, Krasinski A, Punna S, and Watts SW (2016) The adipokine chemerin amplifies electrical field stimulated contraction in the isolated rat superior mesenteric artery. Am J Physiol Heart Circ Physiol 311:H498-H507.

Della Chiesa M, Sivori S, Castriconi R, Marcenaro E, and Moretta A (2005) Pathogeninduced private conversations between natural killer and dendritic cells. Trends Microbiol 13:128-136.

Eaton SL, Roche SL, Llavero Hurtado M, Oldknow KJ, Farquharson C, Gillingwater TH, and Wishart TM (2013) Total protein analysis as a reliable loading control for quantitative fluorescent Western blotting. PLoS One 8:e72457.

Ferland DJ, Darios ES, Neubig RR, Sjögren B, Truong N, Torres R, Dexheimer TS, Thompson JM, and Watts SW (2017) Chemerin-induced arterial contraction is $\mathrm{G}_{\mathrm{i}}$ and calcium-dependent. Vascul Pharmacol 88:30-41.

Garrison RJ, Kannel WB, Stokes J, III, and Castelli WP (1987) Incidence and precursors of hypertension in young adults: the Framingham Offspring Study. Prev Med 16:235-251.

Goralski KB, McCarthy TC, Hanniman EA, Zabel BA, Butcher EC, Parlee SD, Muruganandan S, and Sinal CJ (2007) Chemerin, a novel adipokine that regulates adipogenesis and adipocyte metabolism. J Biol Chem 282:28175-28188.

Hall JE, do Carmo JM, da Silva AA, Wang Z, and Hall ME (2015) Obesity-induced hypertension: interaction of neurohumoral and renal mechanisms. Circ Res 116: 991-1006.

Hashimoto JG, Beadles-Bohling AS, and Wiren KM (2004) Comparison of RiboGreen and 18S rRNA quantitation for normalizing real-time RT-PCR expression analysis. Biotechniques 36:54-56, 58-60.

Hung G, Xiao X, Peralta R, Bhattacharjee G, Murray S, Norris D, Guo S, and Monia BP (2013) Characterization of target mRNA reduction through in situ RNA hybridization in multiple organ systems following systemic antisense treatment in animals. Nucleic Acid Ther 23:369-378.

Mullick AE, Yeh ST, Graham MJ, Engelhardt JA, Prakash TP, and Crooke RM (2017) Blood pressure lowering and safety improvements with liver angiotensinogen inhibition in models of hypertension and kidney injury. Hypertension 70: $566-576$.

Neves KB, Lobato NS, Lopes RA, Filgueira FP, Zanotto CZ, Oliveira AM, and Tostes $\mathrm{RC}$ (2014) Chemerin reduces vascular nitric oxide/cGMP signalling in rat aorta: a link to vascular dysfunction in obesity? Clin Sci (Lond) 127:111-122.

Prakash TP, Yu J, Migawa MT, Kinberger GA, Wan WB, Østergaard ME, Carty RL, Vasquez G, Low A, Chappell A, et al. (2016) Comprehensive structure-activity relationship of triantennary N-Acetylgalactosamine conjugated antisense oligonucleotides for targeted delivery to hepatocytes. J Med Chem 59:2718-2733.

Robinson MW, Harmon C, and O'Farrelly C (2016) Liver immunology and its role in inflammation and homeostasis. Cell Mol Immunol 13:267-276.

Samson M, Edinger AL, Stordeur P, Rucker J, Verhasselt V, Sharron M, Govaerts C, Mollereau C, Vassart G, Doms RW, et al. (1998) ChemR23, a putative chemoattractant receptor, is expressed in monocyte-derived dendritic cells and macrophages and is a coreceptor for SIV and some primary HIV-1 strains. Eur $J$ Immunol 28:1689-1700.

Seth PP, Siwkowski A, Allerson CR, Vasquez G, Lee S, Prakash TP, Kinberger G, Migawa MT, Gaus H, Bhat B, et al. (2008) Design, synthesis and evaluation of constrained methoxyethyl (cMOE) and constrained ethyl (cEt) nucleoside analogs. Nucleic Acids Symp Ser (Oxf) (52):553-554.

Watts SW, Dorrance AM, Penfold ME, Rourke JL, Sinal CJ, Seitz B, Sullivan TJ, Charvat TT, Thompson JM, Burnett R, et al. (2013) Chemerin connects fat to arterial contraction. Arterioscler Thromb Vasc Biol 33:1320-1328.

Wittamer V, Franssen JD, Vulcano M, Mirjolet JF, Le Poul E, Migeotte I, Brézillon S, Tyldesley R, Blanpain C, Detheux M, et al. (2003) Specific recruitment of antigenpresenting cells by chemerin, a novel processed ligand from human inflammatory fluids. J Exp Med 198:977-985.

Zabel BA, Silverio AM, and Butcher EC (2005) Chemokine-like receptor 1 expression and chemerin-directed chemotaxis distinguish plasmacytoid from myeloid dendritic cells in human blood. J Immunol 174:244-251.

Zylla S, Pietzner M, Kühn JP, Völzke H, Dörr M, Nauck M, and Friedrich N (2017) Serum chemerin is associated with inflammatory and metabolic parametersresults of a population-based study. Obesity (Silver Spring) 25:468-475.

Address correspondence to: Dr. David J. Ferland, 1355 Bogue Street, Room B445 Life Sciences, Department of Pharmacology and Toxicology, Michigan State University, East Lansing, MI 48824-1317. E-mail: ferlandd@msu.edu 